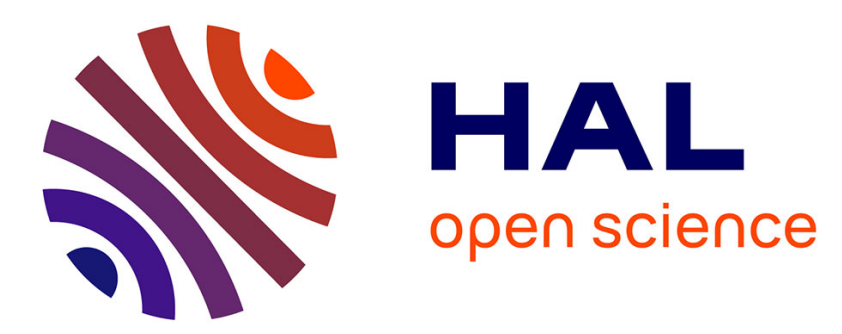

\title{
Exploitation en temps réel de la sonde atomique
}

D. Blavette, A. Bostel, J.M. Sarrau

\section{To cite this version:}

D. Blavette, A. Bostel, J.M. Sarrau. Exploitation en temps réel de la sonde atomique. Revue de Physique Appliquée, 1981, 16 (7), pp.405-409. 10.1051/rphysap:01981001607040500 . jpa-00244933

\section{HAL Id: jpa-00244933 https://hal.science/jpa-00244933}

Submitted on 1 Jan 1981

HAL is a multi-disciplinary open access archive for the deposit and dissemination of scientific research documents, whether they are published or not. The documents may come from teaching and research institutions in France or abroad, or from public or private research centers.
L'archive ouverte pluridisciplinaire HAL, est destinée au dépôt et à la diffusion de documents scientifiques de niveau recherche, publiés ou non, émanant des établissements d'enseignement et de recherche français ou étrangers, des laboratoires publics ou privés. 


\title{
Exploitation en temps réel de la sonde atomique
}

\author{
D. Blavette, A. Bostel et J. M. Sarrau \\ Groupe de Métallurgie Physique (*), Faculté des Sciences de Rouen, B.P. n 67, 76130 Mont Saint Aignan, France
}

(Reçu le 16 février 1981, révisé le 26 mars 1981, accepté le 27 mars 1981)

\begin{abstract}
Résumé. - Un microordinateur assure la gestion et l'exploitation d'une sonde atomique. La masse des ions est calculée à partir de la mesure des temps de vol (à 5 ns près) et des potentiels d'évaporation. Un interface de visualisation permet la construction en temps réel de spectres de masse et la mise en évidence de variations locales de composition chimique d'un alliage. En application, une analyse d'alliage $\mathrm{Fe}-\mathrm{Cr}-\mathrm{Ni}-\mathrm{Al}$ montre la présence de zones riches en $\mathrm{Ni}$ et $\mathrm{Al}$.
\end{abstract}

\begin{abstract}
A computer controlled atom-probe is described. The time of flight and potential measurements give the mass of field evaporated ions. A visualization interface allows us to realize real time mass spectra, and so, to point out the local composition fluctuations. Then a metallurgical application of this apparatus is given. The spectra of a $\mathrm{Fe}-\mathrm{Cr}-\mathrm{Ni}-\mathrm{Al}$ alloy show for instance, the aluminium nickel rich particules presence.
\end{abstract}

1. Principe de la sonde atomique. - La sonde atomique est un microscope ionique associé à un spectromètre de masse à temps de vol. L'échantillon examiné a la forme d'un fil métallique fin dont l'apex présente un très faible rayon de courbure. En appliquant un potentiel continu $V_{0}$ de quelques kilovolts les atomes de la surface de l'échantillon sont soumis à un champ électrique de plusieurs dizaines de volts par nanomètre. Le champ d'évaporation des atomes est atteint en ajoutant à $V_{0}$ un potentiel impulsionnel $V_{\mathrm{p}}$. L'échantillon s'évapore atome par atome, il produit des ions $n$ fois chargés qui traversent un tube de longueur $L$ à une vitesse moyenne $v$. L'état de charge des ions dépend de leur nature, du site cristallographique d'évaporation, du rapport $V_{\mathrm{p}} / V_{0}$ et de l'échantillon analysé. Lors d'une investigation le degré $n$ d'ionisation de chaque espèce est bien défini. En première approximation les ions provenant d'atomes de masse $M$ acquièrent une énergie cinétique $\frac{1}{2} M v^{2}$ correspondant à l'énergie du champ électrique $n e\left(V_{0}+V_{\mathrm{p}}\right)$. $e$ est la charge de l'électron. La mesure du temps $t$ de vol des ions permet de calculer le rapport

$$
\frac{M}{n}=\frac{2 e\left(V_{0}+V_{\mathrm{p}}\right)}{L^{2}} t^{2} .
$$

Une limitation à la résolution en masse de la sonde est la mesure du temps. Elle est faite par des compteurs rapides et est définie à $5 \mathrm{~ns}$ près. La résolution en masse obtenue est de l'ordre de $1 / 300=\Delta M / M$.

(*) E.R.A. 258.
Une analyse chimique de l'échantillon est obtenue en identifiant les ions évaporés. La première sonde atomique a été réalisée par E. W. Mueller [1], depuis d'autres ont été construites $[2,3,4,5,6,7,8,9,10]$.

2. Volume analysé. - L'échantillon se présente sous la forme d'une pointe, il possède un axe de révolution et est approximativement équivalent à un cône d'angle au sommet faible se terminant par une calotte sphérique.

$\mathrm{Au}$ cours de l'évaporation le rayon de courbure de l'apex augmente, le potentiel $V_{0}+V_{\mathrm{p}}$ est alors augmenté pour conserver le champ d'évaporation. L'amplitude de l'impulsion est proportionnelle à $V_{0}\left(V_{\mathrm{p}}=\alpha V_{0}\right)$ de sorte qu'il suffit d'élever progressivement au cours du temps le potentiel $V_{0}$. Le nombre d'impulsions envoyées par seconde et l'évolution de $V_{0}$ fixent le flux moyen d'ions évaporés.

La surface analysée $S_{\mathrm{A}}$ correspond à la surface découpée par la projection stéréographique de la

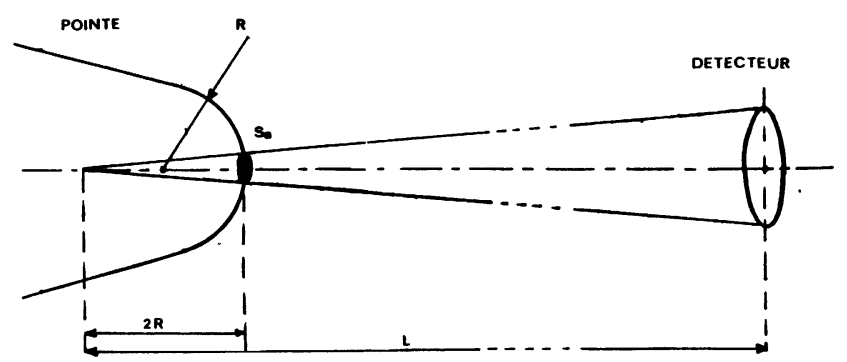

Fig. 1. - Surface analysée.

[Analysis area.] 
surface $S_{\mathrm{D}}$ du détecteur sur l'échantillon (calotte sphérique de rayon de courbure $R$ )

$$
S_{\mathrm{A}} \sim S_{\mathrm{D}} \cdot \frac{4 R^{2}}{L^{2}} \quad \text { (Fig. 1). }
$$

L'analyse est quasi ponctuelle, elle a lieu sur une zone de quelques surfaces de mailles cristallographiques élémentaires.

3. Les résultats et leur exploitation. - Les données acquises et relatives à la réception d'un ion sont : le temps de vol $t$, le potentiel d'évaporation $V_{0}(1+\alpha)$, le numéro $N$ de l'impulsion d'évaporation.

$N$ correspond au nombre d'impulsions appliquées à l'échantillon depuis le début de l'analyse. Il représente à tout instant le volume de matière exploré depuis le début de l'investigation.

Une analyse est constituée d'une suite de données $\left(t, V_{0}, N\right)$. A partir de ces résultats divers modes de représentation sont possibles :

1) Le calcul et le classement des masses conduisent à la formation d'un spectre de masse représentatif de la composition chimique de la zone explorée.

L'analyse est totale et globale si elle regroupe tous les résultats d'une expérimentation. Elle est partielle et locale si elle est faite sur un petit nombre de résultats.
Elle correspond alors à une portion de matière de l'échantillon. Dans ce mode de représentation la notion d'ordre d'arrivée des atomes (c'est-à-dire de leur distance relative) est perdue.

2) Des résultats fragmentaires peuvent être extraits de l'ordre d'arrivée des ions. En effet, lörsque la cadence d'évaporation est régulière, le flux d'ions reçus est modulé par le passage des plans atomiques successifs de l'échantillon. Il en résulte que la courbe caractérisant le nombre d'ions reçus en fonction du nombre d'impulsions appliquées présente une succession d'accroissements équivalents à des marches [11]. Dans ce cas, la surface analysée ne doit pas comprendre plusieurs bords de plans atomiques. Cette modulation est aussi détectée lorsque l'évaporation a lieu en présence de gaz image, par la variation du courant d'ions gazeux reçus sur le détecteur [12].

Il est ainsi possible de suivre l'évolution d'une espèce atomique en fonction du nombre de plans évaporés et de déterminer la profondeur d'analyse. Cela conduit à l'évaluation d'une concentration locale [13].

4. Gestion de la sonde. - L'expérience est gérée par un séquenceur et un micro-ordinateur type AIM 65 (Fig. 2) dont l'unité centrale est un microprocesseur 8 bits (6502).

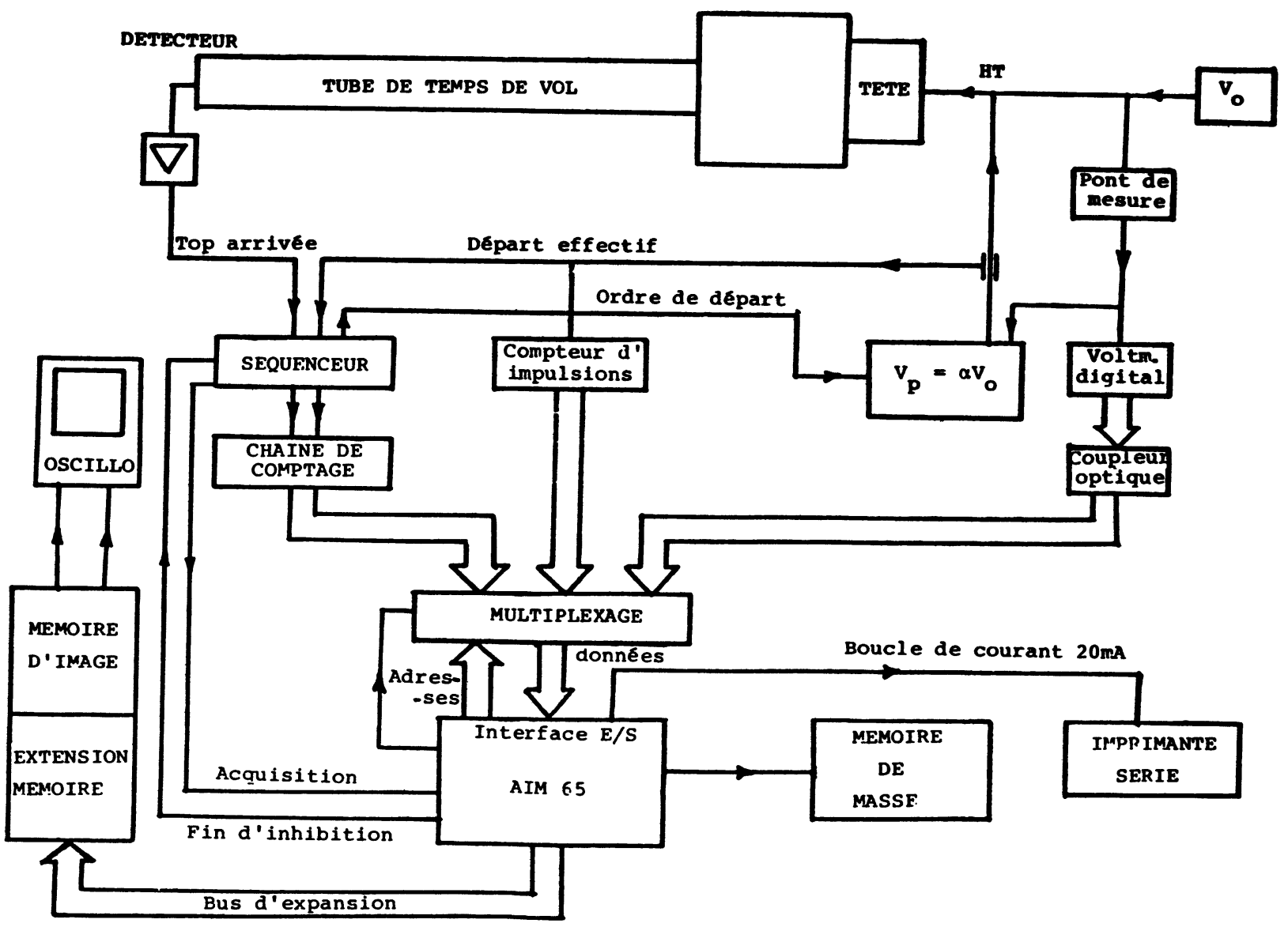

Fig. 2. - Schéma fonctionnel de la sonde atomique.

[Atome probe configuration.] 
Le séquenceur donne un ordre de départ au générateur d'impulsions, puis le signal de départ effectif de l'ion déclenche trois compteurs de mesure du temps. L'arrivée des ions successifs sur le détecteur arrête l'un après l'autre ces compteurs. L'expérience montre que la probabilité de recueillir quatre ions à partir d'une même impulsion est extrêmement faible.

Si après un temps de vol maximum $t_{\mathrm{m}}(24 \mu \mathrm{s}$ pour $L=2 \mathrm{~m}$ ) aucun ion n'est détecté, le séquenceur répète le processus, la cadence est de 10 impulsions par seconde. $\mathrm{Si}$ au contraire, un ou plusieurs ions sont détectés pendant le temps $t_{\mathrm{m}}$, un ordre d'acquisition est envoyé à l'AIM 65. Le micro-ordinateur stoppe le séquenceur. Les temps de vol, le potentiel continu, le numéro d'impulsion sont alors mis en mémoire. Après traitement l'AIM autorise la poursuite de la manipulation.

5. Saisie et traitement des données. - Le grand nombre de résultats $\left(\gg 10^{3}\right)$ par expérimentation nécessite leurs représentations sous forme synthétique. Il est souhaitable de mettre en place des aides à l'interprétation des résultats qui modifient selon l'état actuel de l'expérience, certains paramètres expérimentaux ou de calcul. Des logiciels de traitement en temps réel procurent une interactivité avec la manipulation. Une interface spécialisée dans la visualisation a été prévue à cet effet.

Les programmes de saisie et de traitement ont été développés sur l'AIM 65 même. Les aides au développement, tels que les langages basic et assembleur ( 2 passes), résidant en mémoire morte, facilitent grandement la programmation [14]. Le programme basic utilise des routines assembleur qui assurent la gestion fine de type électronique : acquisition, démultiplexage formatage, stockage des données, scrutation clavier. Le logiciel basic est réservé aux calculs et à la mise à jour de là visualisation [15].

Les données $\left(t, V_{0}, N\right)$ sont stockées directement dès leurs arrivées sur une mémoire de masse pour être définitivement conservées et exploitées ultérieurement. Les masses sont aussi calculées au fur et à mesure de l'arrivée des données et sont envoyées sur une imprimante par l'intermédiaire d'une boucle de courant $20 \mathrm{~mA}$. Une suite des ions reçus est ainsi obtenue pendant toute la durée de l'expérimentation.

Lors de la réception d'un ion, le logiciel assembleur prend le contrôle des adresses de multiplexage des données. Le démultiplexage des données, assuré par programme, conduit au stockage sur cassette et au formatage des données en code ASCII. Le programme principal basic exploite alors les données sous cette forme et actualise la mémoire de visualisation. Le programme assembleur reprend alors le contrôle et autorise une nouvelle acquisition. Pendant le temps où aucune acquisition n'est demandée, le logiciel assembleur effectue une scrutation du clavier de l'AIM 65.

Un certain nombre de touches donne l'accès à des routines de traitements spécialisés. L'utilisateur intervient alors sur les paramètres de la manipulation et. son exploitation.

A tout instant, il est possible d'effacer la mémoire d'image, changer ses caractéristiques, clore le fichier de stockage des données, inscrire des commentaires au sein de la liste de résultats, éditer des spectres de masse.

L'implémentation de nouvelles fonctions est créée selon les besoins; le jeu des fonctions disponibles apparait alors comme une véritable aide à l'interprétation et à la décision.

6. Description de l'interface de visualisation. - La mémoire d'image se comporte vis-à-vis de l'unité centrale comme n'importe quelle portion de son espace adressable (Fig. 3). Son accès est aisé et ne nécessite aucune instruction spécialisée. Un organe de gestion des entrées-sorties assure la transparence des accès mémoire. Le bus de contrôle du microprocesseur est utilisé par la logique d'arbitrage des bus locaux (de données et d'adresses). En dehors des rares accès à la mémoire d'image, un générateur d'adresses propre à l'interface d'image balaie les lignes d'adressage du système. Un convertisseur digital-analogique (D.A.) connecté au bus de données assure la génération d'un signal analogique récurrent.

Lors d'un accès, les lignes d'adresses et de données se déconnectent du générateur d'adresses, et du convertisseur D.A. afin que l'unité centrale accède à ces deux bus. Les bus de données et d'adresses sont donc multiplexés. La logique de gestion des entréessorties définit les signaux d'écriture, de sélection des différents organes de l'interface.

L'application du signal analogique à un oscilloscope visualise une courbe sous forme échantillonnée. La taille de la mémoire d'image limite la résolution à $1 / 256$. Un signal de synchronisation est produit, à chaque balayage de mémoire, pour assurer la stabilité de l'image.

L'utilisation de la base de temps retardée de l'oscilloscope offre la possibilité d'examiner avec plus de précision une partie de l'image. La modification de l'échelle n'interagit pas avec le logiciel et reste transparente pour le système.

Des spectres de masse sont ainsi formés en temps réel. L'investigation de l'échantillon est suivie par l'évolution des différents pics de masse en cours d'analyse. Une édition de ces spectres est possible à tout instant. La manipulation est ainsi orientée en fonction des résultats obtenus (changement du site d'analyse).

7. Application à l'analyse d'un alliage $\left({ }^{1}\right)$. - Les figures $4 \mathrm{~A}$ et $4 \mathrm{~B}$ montrent l'allure de deux spectres partiels tirés au cours d'une analyse d'un alliage de fer,

( ${ }^{1}$ ) Nous remercions M. Thomas (IRSID) de nous avoir fourni l'alliage et des fructueuses discussions que nous avons pu avoir avec lui. 


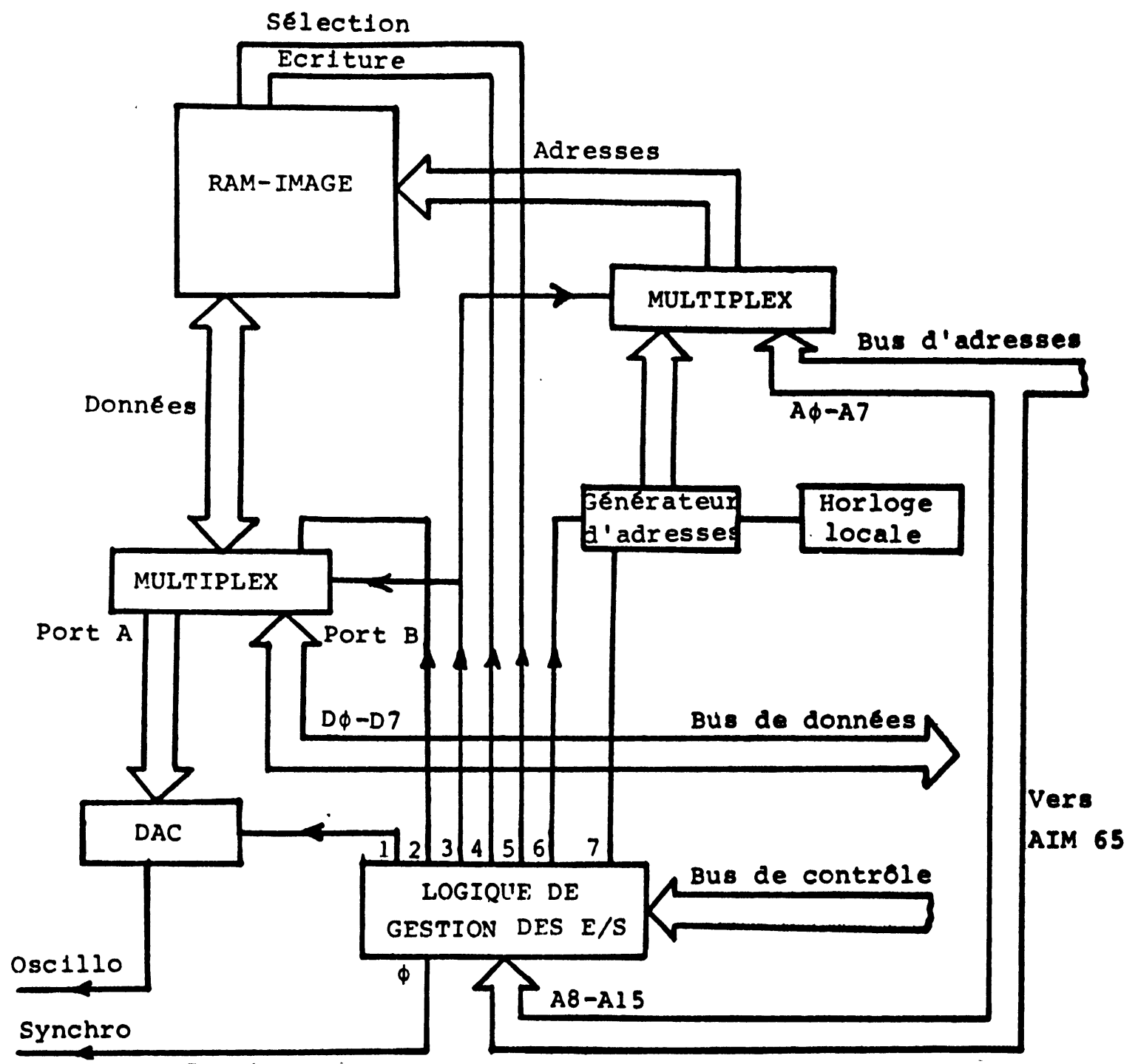

Fig. 3. - Schéma de l'interface de visualisation. 0 Synchronisation de l'oscillo. 1 Inhibition de la conversion digitale. 2 Lecture/écriture du multiplexeur 3 états. 3 Contrôle du multiplexage. 4 Lecture/écriture de la mémoire. 5 Sélection page mémoire. 6 Bloquage du générateur d'adresses. 7. Détection du cycle «balayage mémoire».

[Visualization interface scheme.]

chrome, nickel, aluminium. L'alliage présente après un recuit de $17 \mathrm{~h}$ à $550^{\circ}$, un durcissement structural lié à la précipitation de phases intermétalliques. La taille des précipités étant de 50 à $100 \AA$, la sonde atomique est particulièrement adaptée à l'analyse de la composition de ces précipités. La figure 4A met en évidence une zone de composition différente de la matrice (Fig. 4B). Une remise à zéro de la mémoire d'image a été effectuée après l'édition du spectre $4 \mathrm{~A}$ pour obtenir ensuite le spectre 4B.

Le spectre de la figure $4 \mathrm{~A}$ montre une présence importante d'ions ionisés deux fois d'aluminium (pic 13,5) et de nickel (pic 29) par rapport au spectre de la figure 4B. Ces deux spectres partiels successifs correspondent environ à un même nombre total d'ions et montrent bien la présence d'une zone riche en aluminium et en nickel dans l'alliage [16]. Ces deux spectres correspondent à la copie sur imprimante des images visualisées lors de l'investigation. L'analyse globale de l'échantillon porte sur 1200 ions et conduit à un spectre ayant l'allure du spectre $4 \mathrm{~B}$ confirmant la concentration nominale de l'alliage ( $\mathrm{Fe} 76 \%$, Cr $20 \%$, Ni $2 \%$, Al $2 \%$ ).

L'utilisation d'un micro-ordinateur à faible coût interfacé à la sonde à atomes facilite l'exploitation des résultats. En particulier, l'évolution en cours d'analyse de la composition chimique d'un alliage est contrôlée. Grâce à sa résolution atomique, la sonde permet de définir les variations locales de concentrations au niveau de l'interface précipitématrice d'un alliage.

La mise en place d'un système d'exploitation efficace des résultats de la sonde atomique permet d'en faire un outil puissant dans le domaine de la métallurgie des alliages. 

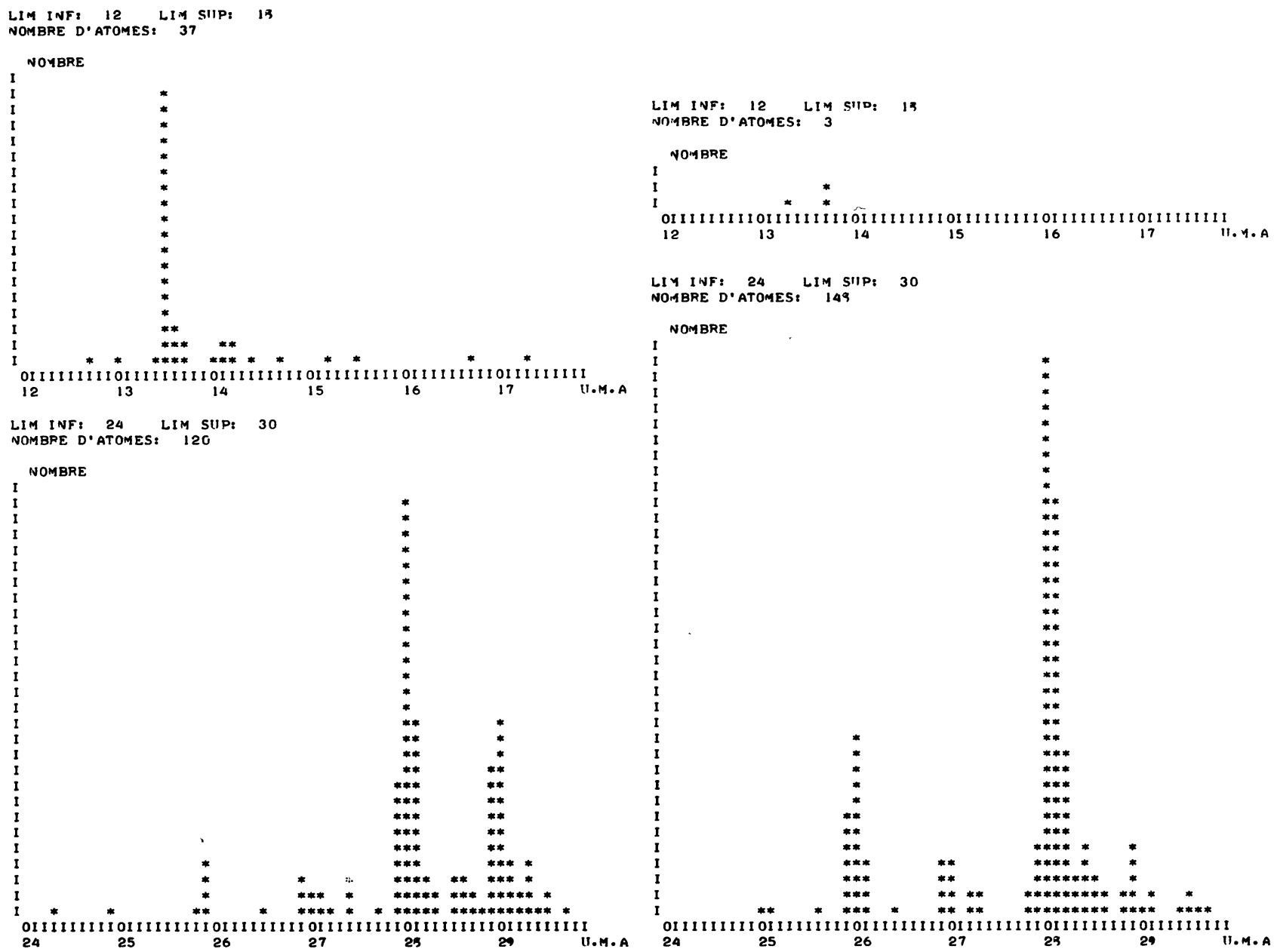

Fig. 4A. - Spectre d'un alliage de Fe-Cr-Ni-Al.

Fig. 4B. - Spectre d'un alliage de Fe-Cr-Ni-Al.

[A Fe-Cr-Ni-Al alloy spectrum.]

[A Fe-Cr-Ni-Al alloy spectrum.]

\section{Bibliographie}

[1] Muller, E. W., Panitz, J. A., Mac Lane, S. B., Rev. Sci. Instrum. 39 (1968) 83.

[2] Johnson, C. A., Rev. Sci. Instrum. 41 (1970) 12.

[3] Berger, A. S., Rev. Sci. Instrum. 44 (1973) 5.

[4] Brenner, S. S., MC Kinney, J. T., Surf. Sci. 23 (1970) 88.

[5] Turner, P. J., Southon, M. J., Dynamic Mass Spect., Eds D. Price et al. (Heyden, London) 1970.

[6] Panitz, J. A., Rev. Sci. Instrum. 44 (1973) 1034.

[7] Gallot, J., Sarrau, J. M., Bostel, A., Le Vide 30 (1975) 73.

[8] Hall, T. M., Wagner, A., Berger, A. S., Seidman, D. N., Scr. Metall. 10 (1976) 485.

[9] Lewis, R. J., Smith, G. D. W., Proc. Decus Conference, The Hague, Netherland 1 (1975) 705.
[10] Kinoshita, K., Nakamura, S., Kuroda, T., Jpn. J. Appl. Phys. 14 (1975) 2083.

[11] Yamamoto, M., Seidman, D. N., Nakamura, S., 27th Inter. Field Symp., Tokyo, Japan (1980) 317.

[12] Sarrau, J. M., Martin, C., Bostel, A., Gallot, J., $C$. $R$. Hebdh Séan. Acad. Sci. t. 290 (1980) B. 63.

[13] Hall, T. M., Wagner, A., Seidman, D. N., J. Phys. E. 10 (1977) 883.

[14] ZAKs, R., (1979) Programmation du 6502 Sibex.

[15] ARSAC, J., La construction de programmes structurés (Dunod) (1977).

[16] BLAVETTE, D. et al., (à paraître) 